\title{
A microbiological study of neonatal conjunctivae and conjunctivitis
}

\author{
M. J. PRENTICE, G. R. HUTCHINSON, AND D. TAYLOR-ROBINSON \\ From the Division of Communicable Diseases, MRC Clinical Research Centre, and Northwick Park \\ Hospital, Watford Road, Harrow, Middlesex
}

SUMMARY To investigate the importance of chlamydiae, ureaplasmas, Mycoplasma hominis, and anaerobic bacteria in the pathogenesis of neonatal conjunctivitis in the Harrow population conjunctival specimens from 104 infants with conjunctivitis and 104 similar healthy neonates were examined. The incidence of neonatal conjunctivitis was $8.2 \%$, and no case of neomycin-resistant disease occurred during the study. Staphylococcus aureus, viridans Streptococci, and Escherichia coli were the only micro-organisms isolated significantly more frequently from affected than from control eyes, which suggests that these bacteria may be a cause of the conjunctivitis. All cultures for chlamydiae, M. hominis, Neisseria gonorrhoeae, and anaerobic bacteria were negative. The mother's race, social status, illness, and obstetric events were found to have no effect on the incidence, time of onset of conjunctivitis, or micro-organisms isolated. The clinical characteristics of conjunctivitis were also not related to the micro-organisms isolated. No potential pathogens were isolated from $63.5 \%$ of the eyes showing conjunctivitis. The results suggest that some of these cases may be caused by chemical irritation, and the possibility of an infectious aetiology is also discussed.

Neonatal conjunctivitis is a common disease affecting between $2.6 \%$ (Watson and Gairdner, 1968 ) and 5 to $8 \%$ (Hurley, 1966) of infants born in England. The inflammation usually resolves spontaneously within a few days and so investigation of the aetiological agent rarely extends beyond routine bacterial culture. This may occasionally reveal Neisseria gonorrhoeae infection, which needs urgent treatment to prevent progression of the disease. However, Chlamydia trachomatis infections should also be considered. Although these may resolve spontaneously, if untreated they may occasionally result in corneal scarring, which may lead to pannus formation (Freedman et al., 1966; Thygeson, 1971).

An infective agent causing neonatal conjunctivitis is most likely to be inoculated during vaginal delivery of the infant. Pathogens of the disease should therefore be sought among components of the vaginal flora in pregnancy (de Louvois et al., 1975 ) and among aetiological agents of vaginitis or cervicitis. C. trachomatis has been isolated from the cervices of apparently asymptomatic women (Oriel

Address for reprints: Dr D. Taylor-Robinson, Division of Communicable Diseases, MRC Clinical Research Centre, Watford Road, Harrow, Middx et al., 1974; Burns et al., 1975), but some cases of cervicitis may be caused by this organism (Chiang et al., 1968; Hobson et al., 1976). The evidence for this, however, is less conclusive than it is in the case of non-specific urethritis in men (Dunlop et al., 1965; Schachter et al., 1975; Bowie et al., 1976). There is also evidence to suggest that some strains of Ureaplasma urealyticum (ureaplasmas) may cause urethritis (Prentice et al., 1976; Bowie et al., 1976; Taylor-Robinson et al., 1977), and it is possible that ureaplasmas could also be a cause of neonatal conjunctivitis. Jones and Tobin (1968) found Mycoplasma hominis in $3.5 \%$ of eyes of neonates with conjunctivitis, but infants carrying ureaplasmas or $\boldsymbol{M}$. hominis in the nose, throat, umbilical or genital regions have not had an increased incidence of conjunctivitis (Klein et al., 1969; Foy et al., 1970). There has, however, been no report of the incidence of ureaplasmas on healthy or diseased neonatal conjunctivae, nor has the incidence of chlamydial neonatal conjunctivitis been recorded by use of the McCoy cell technique of isolation.

Over a period of 8 months an attempt was made in a maternity unit to isolate chlamydiae, ureaplasmas, $M$. hominis, and bacteria, including anaerobic species, from the eyes of all neonates with conjunc- 
tivitis and from healthy control infants in order to study the role of these micro-organisms in the disease.

\section{Materials and methods}

\section{PATIENTS}

Consecutive cases of untreated neonatal conjunctivitis occurring between February and September 1975 in the maternity unit at Northwick Park Hospital were examined. Swabs were taken from those infants who developed purulent conjunctival exudate, and control samples were taken from unaffected neonates on the same ward, matched for age, mode of delivery, and when possible for systemic antibiotic treatment of mother or baby. Permission to take specimens was always given by the mother, to whom information about the study was freely available. The project was approved by the Northwick Park Hospital Ethical Committee.

\section{STUDY DESIGN AND COLLECTION OF SPECIMENS}

Samples were taken from both eyes of 104 infants with conjunctivitis and from either the left or right eye of 104 controls, the side sampled corresponding to that involved in the affected baby. To avoid cross-contamination swabs were taken from infants without disease before handling any babies with conjunctivitis. Swabs were not taken while the babies were crying.

The amounts of conjunctival exudate, inflammation of the eyelids, and periorbital oedema were recorded. The conjunctiva was exposed by gentle traction on the eyelid and the following specimens were taken:

(a) For bacterial culture, a small $(1 \mu \mathrm{l})$ disposable plastic bacteriological loop (NUNC) or a fine platinum wire loop was swept over the conjunctiva and immediately streaked out over a chocolate and a blood agar plate. Care was taken to avoid contamination from the skin and eyelashes. A second, similar loop was used to inoculate a 'GC selective agar medium' plate (Oxoid), which was placed in a candle jar. In addition, in 63 out of 104 cases, this loop was first used to inoculate a prereduced blood agar plate, which was immediately sealed in a McIntosh and Fieldes jar containing a new 'GasPak' (Becton, Dickinson, and Co).

(b) For mycoplasmal culture a calcium alginate swab was rolled over a portion of conjunctiva and then agitated in $1.5 \mathrm{ml}$ of mycoplasma liquid medium.

(c) For chlamydial culture a cotton-wool-tipped ear, nose, and throat swab was drawn firmly over the greatest possible area of conjunctiva and then energetically agitated in $0.8 \mathrm{ml}$ of sucrose-phosphate transport medium (Darougar et al., 1970) containing $10 \%$ fetal calf serum. This was plunged into liquid nitrogen within 5 minutes.

(d) To examine for trichomonads conjunctival exudate was taken with a loop from 32 patients, and a wet preparation was made with saline on a microscope slide. The edges of the coverslip were sealed with nail varnish. Because samples for culture were given priority, smears for trichomonads were made either with exudate already outside the conjunctival sac or after all other specimens had been taken.

The conjunctivitis was treated with local neomycin by the paediatricians if they thought it necessary, and, if not, the affected eyes were swabbed with sterile saline. Neomycin was chosen because it does not affect the growth of chlamydiae. Neomycin-resistant conjunctivitis could then be identified, diagnosed, and, if chlamydial, thoroughly treated with tetracycline ointment.

The infants who had conjunctivitis were observed daily as long as they were in hospital. The district midwives, who visit $76 \%$ of babies born in the hospital for up to 2 weeks after delivery, were asked to seek and report cases of conjunctivitis throughout the period of the study. During that time the total number of neonates who developed conjunctivitis in hospital or at home was recorded.

Information about the mothers and infants in the study was collected. This included details of age, race, marital status, occupation, past history of infections and medication, complications of this and any previous pregnancy, the mode of delivery, the condition of the infant, puerperal infections and treatment, and also whether breast or bottled milk was given.

\section{LABORATORY PROCEDURES}

The chocolate/blood agar plates were incubated in an atmosphere of $5 \% \mathrm{CO}_{2}$ and, like all the bacterial cultures, at $37^{\circ} \mathrm{C}$. The chocolate and aerobic blood agar plates were observed at 24 and 48 hours after inoculation. Thirty of the 63 anaerobic agar plates were incubated for 2 days and the remainder for 3 days before the jars were opened. The procedures necessary to identify any bacterial growth were performed by the staff of the Microbiology Department of Northwick Park Hospital. The gonococcal selective agar plates were incubated in candle jars, examined at 24 and 48 hours, and any colonies were tested with fresh oxidase reagent.

The methods of isolating Chlamydia trachomatis, Ureaplasma urealyticum, and Mycoplasma hominis were as previously described (Prentice et al., 1976).

The wet smears for trichomonads were examined 
under dark ground illumination within two hours of preparation.

STATISTICAL ANALYSIS

The probability of obtaining a positive bacterial isolation was estimated, with standard errors, for each organism and for each of the following four categories when one eye was chosen at random: (a) bilateral conjunctivitis, $(b)$ affected eye in unilateral conjunctivitis, $(c)$ unaffected eye in unilateral conjunctivitis, and $(d)$ no conjunctivitis-controls.

Fisher's exact probability test was applied with one degree of freedom in comparing the isolation of micro-organisms from conjunctivitis cases which occurred within $24 \mathrm{hrs}$ of delivery and those which occurred after the first day of life (Table 1).

Results were considered significant if $P \leqslant 0 \cdot 05$.

\section{Results}

INCIDENCE OF CONJUNCTIVITIS

Satisfactory specimens were obtained from 104 affected neonates, though 128 cases of conjunctivitis occurred in hospital during the study period. An additional 35 new cases beginning at home were reported by district midwives. Since only $76 \%$ of infants were discharged from hospital into the area visited by the midwives, the expected number of cases of conjunctivitis occurring in infants after leaving hospital is 47 . The 128 cases in hospital and 47 estimated at home results in a total of 175 babies affected out of 2142 infants delivered in that period. The incidence of neonatal conjunctivitis in the Harrow area is therefore estimated to be $8.2 \%$.

\section{MICRO-ORGANISMS ISOLATED}

The conjunctivitis was present in one eye in 59 neonates and in both eyes in 45 neonates. Specimens were therefore taken from 149 eyes showing conjunctivitis, 59 normal-looking opposite eyes of affected babies, and 104 eyes of healthy control infants. The micro-organisms isolated from these three groups are listed in order of frequency in Table 2.

Staphylococcus epidermidis was the organism isolated most frequently from all groups, but the
Table 2 Micro-organisms isolated

\begin{tabular}{|c|c|c|c|}
\hline Micro-organism & $\begin{array}{l}\text { Eyes with } \\
\text { conjunctivitis } \\
\text { (149) }\end{array}$ & $\begin{array}{l}\text { Normal- } \\
\text { looking } \\
\text { opposite eyes } \\
\text { of patients with } \\
\text { conjunctivitis } \\
(59)\end{array}$ & $\begin{array}{l}\text { Control } \\
\text { eyes } \\
\text { (104) }\end{array}$ \\
\hline $\begin{array}{l}\text { Staph. epidermidis } \\
\text { Staph. epidermidis } \\
\quad \text { (alone) } \\
\text { Viridans Streptococcus } \\
\text { Staph. aureus } \\
\text { E. coli } \\
\text { U. urealyticum } \\
\text { Pseudomonas } \text { sp. } \\
\text { Klebsiella } \text { sp. } \\
\text { Proteus sp. } \\
\text { Neisseria } \text { sp. } \\
\text { Corynebacterium } \\
\text { ulcerans } \\
\text { Diphtheroids } \\
\text { Str. pneumoniae } \\
\text { Non-haemolytic Strep. } \\
\text { sp. } \\
\text { Micrococcus sp. } \\
\text { Aerobic spore-former } \\
\text { No isolate }\end{array}$ & $\begin{array}{l}31(21 \%) \\
(15) \\
25(16 \%) \\
12(8 \%) \\
8(5 \cdot 4 \%) \\
5(3.4 \%) \\
2 \\
2 \\
-1 \\
1 \\
2 \\
1 \\
1 \\
1 \\
80(53.5 \%)\end{array}$ & $\begin{array}{l}13(22 \%) \\
(10) \\
4(6 \cdot 8 \%) \\
5(8 \cdot 5 \%) \\
- \\
- \\
- \\
- \\
- \\
- \\
- \\
- \\
39(66 \%)\end{array}$ & $\begin{array}{l}33(32 \%) \\
(28) \\
3(2.9 \%) \\
3(2.9 \%) \\
1(1 \%) \\
2(1.9 \%) \\
- \\
- \\
- \\
- \\
- \\
- \\
- \\
65(62.5 \%)\end{array}$ \\
\hline $\begin{array}{l}\text { Any micro-organism } \\
\text { isolated } \\
\text { Any micro-organism } \\
\text { isolated, excluding } \\
\text { Staph. epidermidis }\end{array}$ & $69(46 \%)$ & $20(34 \%)$ & $39(37.5 \%)$ \\
\hline
\end{tabular}

isolation rate in the conjunctivitis group $(21 \%)$ was not significantly different $(P>0.05)$ from that in the control groups (32\% and $22 \%)$.

Viridans streptococcus was isolated from 25 $(16 \%)$ eyes showing conjunctivitis, $4(6.8 \%)$ opposite eyes, and $3(2.9 \%)$ control conjunctivae. The difference in isolation rates for the affected and control eyes was significant whether the disease was unilateral $(P<0.01)$ or bilateral $(P<0.05)$. In addition the difference between the isolation rates for the affected eyes and the opposite eyes was significant $(P<0.05)$, but was not significant for the opposite eyes when compared with the controls $(\mathrm{P}>0.05)$.

Staphylococcus aureus was isolated from 12 eyes showing conjunctivitis, 5 opposite eyes and 3 control eyes. The isolation rate in the conjunctivitis group was significantly different from that in the

Table 1 Relationship between the infants' age at the onset of disease and the presence of micro-organisms

\begin{tabular}{lcccc}
\hline Age at onset of conjunctivitis & No. of patients & $\begin{array}{l}\text { No. of eyes with } \\
\text { conjunctivitis }\end{array}$ & $\begin{array}{l}\text { No. of eyes with no } \\
\text { micro-organisms } \\
\text { isolated }\end{array}$ \\
\hline 0-12 hours & 4 & 8 & 8 & $\begin{array}{l}\text { No. of eyes with any } \\
\text { micro-organisms } \\
\text { isolated }\end{array}$ \\
13-23 hours & 5 & 9 & 9 & 0 \\
1-2 days & 43 & 64 & 38 & 26 \\
3-6 days & 44 & 56 & 20 & 5 \\
$>6$ days & 8 & 12 & 5 & 7 \\
\hline
\end{tabular}

*Significant difference in isolations before and after $24 \mathrm{~h}(\mathrm{P}<0.006)$. 
control group $(\mathrm{P}<0.05)$ when the disease was unilateral but not when bilateral. When the isolation rate of the affected group was compared with that for unaffected opposite eyes, there was no significant difference $(P>0.05)$.

Escherichia coli was isolated from 8 eyes showing conjunctivitis and from 1 control eye. This difference in isolation rate was significant $(\mathrm{P}<0.05)$. E. coli was not isolated from any of the unaffected opposite eyes.

No other micro-organism was isolated frequently enough to permit statistical analysis. No bacteria grew under anaerobic conditions if they did not appear in the aerobic media. Neisseria gonorrhoeae, Mycoplasma hominis, and Chlamydia trachomatis were not isolated from any patient, nor was Trichomonas vaginalis seen in any specimen examined.

More than one species of micro-organism were isolated on 21 occasions from eyes with conjunctivitis, on 4 occasions from unaffected opposite eyes, and on 5 occasions from control eyes. In the majority of cases Staph. epidermidis was the other organism in the combination, being isolated with another micro-organism on 15 occasions from diseased eyes, on 3 occasions from unaffected opposite eyes, and on 5 occasions from control eyes.

Since Staph. epidermidis is usually a skin commensal, the number of times this bacterium was isolated alone was subtracted from the results (Table 2). The frequency of isolation of micro-organisms from eyes with conjunctivitis was then found to be greater than from opposite eyes of the affected infants $(P<0.01)$ or from control eyes $(P<0.01)$. These differences in the frequency of isolation could be due to differences between subjects. However, this is not likely because there was no significant difference between the frequency of isolation of micro-organisms from opposite eyes of affected infants and from control eyes.

ONSET, SIGNS, AND DURATION OF

CONJUNCTIVITIS

(1) Neonatal age at onset. Half of the cases of conjunctivitis began within the first 48 hours of life. No micro-organisms were isolated from any of the 9 neonates who developed conjunctival exudate within 24 hours of delivery (Table 1). In this respect these 9 infants are significantly different $(P<0.006)$ from those whose disease started after the first day of life. The fact that 8 of the 9 infants had bilateral conjunctivitis was also significant $(P<0.02)$. The signs and duration of conjunctivitis, however, were similar irrespective of time of onset. Furthermore, following the first day after birth, the types and incidence of micro-organisms isolated were also unrelated to the time of onset of the disease.
(2) Signs of conjunctivitis. The amounts of inflammation of the eyelids, periorbital oedema, and conjunctival exudate were subjectively graded into 4 categories according to severity. None of these categories was particularly associated with the presence of a micro-organism, nor was it related to the time of onset of disease.

Seven healthy control infants subsequently developed conjunctivitis between 2 and 7 days after the swabs were taken. The disease occurred in the eye previously swabbed in 4 babies and in the opposite eye in the other 3 . When these babies were swabbed again, the bacteria isolated did not correspond to those isolated from the eyes of the original affected babies for which the former infants were controls.

(3) Duration and response to treatment. The observed duration of conjunctivitis is shown in Table 3. Two days after delivery $36 \%$ of all babies born in the hospital were discharged home, and therefore only $64(61.5 \%)$ of the patients were watched until their signs disappeared. This occurred within 6 days of onset in about $80 \%$ of the cases, whether or not treatment was given. In one neonate of 27 weeks' gestation signs persisted for more than 2 weeks. Staph. aureus was isolated, and the conjunctivitis was treated with neomycin for a week. Two days later the exudate recurred and Staph. aureus was again isolated. After a further 6 days of neomycin therapy the signs finally resolved. No case of neomycin-resistant conjunctivitis was seen during this study.

\section{EFFECT OF MATERNAL FACTORS ON} CONJUNCTIVITIS

(1) Race. $79 \cdot 8 \%$ of the mothers of babies with conjunctivitis and $77.9 \%$ of the control group were Caucasian. Of the remainder, $12.5 \%$ were of Asian origin in both groups, and $7.7 \%$ of the conjunctivitis group and $9.6 \%$ of the control group comprised mainly West Indian or African negroes. The incidence and types of micro-organisms isolated

Table 3 Duration of disease and effect of treatment

\begin{tabular}{|c|c|c|c|}
\hline Duration (days) & $\begin{array}{l}\text { Total no. of } \\
\text { patients }\end{array}$ & $\begin{array}{l}\text { No. of } \\
\text { untreated } \\
\text { patients }\end{array}$ & $\begin{array}{l}\text { No. treated } \\
\text { with } \\
\text { neomycin }\end{array}$ \\
\hline $\begin{array}{l}<2 \\
3-4 \\
5-6 \\
7-8 \\
9-10 \\
11-12 \\
14+ \\
\text { Unknown } \\
\text { Total }\end{array}$ & $\begin{array}{r}7 \\
24 \\
19 \\
8 \\
2 \\
3 \\
1 \\
40 \\
104\end{array}$ & $\begin{array}{r}7 \\
9 \\
12 \\
6 \\
0 \\
1 \\
0 \\
30 \\
65\end{array}$ & $\begin{array}{r}0 \\
15 \\
7 \\
2 \\
2 \\
2 \\
1 \\
10 \\
39\end{array} 76 \% *$ \\
\hline
\end{tabular}


from the non-Caucasian babies were similar to those isolated from the Caucasian babies.

(2) Marital and social status. The mothers of 8 $(7 \cdot 7 \%)$ infants with conjunctivitis and the same number of mothers of infants in the control group were unmarried. The age of these infants at the onset of the disease and the incidence and types of micro-organisms isolated were no different from those of the babies of married mothers.

The proportion of parents in social classes I and II with affected infants was $32.5 \%$, which is similar to that of the healthy babies, $38.5 \%$.

(3) Maternal disease and antibiotic treatment. A past history of urinary tract infection was given by the mothers of 20 affected and 19 healthy neonates. The types and incidence of micro-organisms in the conjunctival specimens from these infants were no different from the rest of the population studied. $E$. coli was isolated from only 1 baby with conjunctivitis whose mother had had urinary tract infection. The results were also similar whether or not the mothers had been pyrexial since their delivery or had taken antibiotics during the pregnancy or in the puerperium.

(4) Amniocentesis and mode of delivery. Although amniocentesis may provide an opportunity for the introduction of infection, the onset of conjunctivitis was no earlier in the 19 infants whose mothers had had this procedure performed during the pregnancy. Furthermore, the micro-organisms isolated from this group were similar to those isolated from the rest of the infants examined.

The composition of the conjunctival flora in infants who developed conjunctivitis after a difficult delivery, the use of forceps, or a delivery delayed more than 24 hours after rupture of the fetal membranes was similar to that seen in babies delivered with ease. The micro-organisms isolated from the conjunctivae of the 12 affected infants born by caesarian section were also no different from those in the eyes of the infants born by vaginal delivery.

OTHER FACTORS AFFECTING CONJUNCTIVITIS Neither the gestational age of the infants, nor the presence of coexisting disease bore any relation to the micro-organisms isolated. The weights of $26 \%$ of the infant population studied were below the 50th centile for gestational age, and ureaplasmas were isolated from only 1 infant in this low-birthweight group.

Breast milk was fed to $63 \%$ of the patients and to $75 \%$ of the healthy infants examined. $E$. coli was isolated from the eyes of 1 healthy breast-fed baby and 6 infants with conjunctivitis. Four of the latter were bottle-fed, and information on feeding was not available for the remaining 2 infants.

\section{Discussion}

Despite our ability to isolate chlamydiae from up to $47 \%$ of men suffering from non-specific urethritis, in the present study chlamydiae or gonococci were not isolated from conjunctival specimens of 104 neonates with conjunctivitis. This is in contrast to the results of some other workers. Thus, Sowa et al. (1968) cultured chlamydiae in yolk sacs from $4 \%$ of affected neonates in the Gambia, where trachoma is endemic. Hansman (1969) diagnosed chlamydial infection in $3 \%$, Watson and Gairdner (1968) in 9\%, and Armstrong et al. (1976) in $28.5 \%$ of neonatal conjunctivitis cases. Chlamydiae were detected in the latter three studies despite use of the relatively insensitive technique of cytological examination of conjunctival scrapings. The McCoy cell culture technique, however, has been shown to be at least twice as sensitive as cytological examination (Darougar et al., 1970) and also more sensitive than yolk sac isolation (Gordon et al., 1969). In Liverpool, Rees and Hobson (1974) used McCoy cells and isolated chlamydiae from $32 \%$ of a selected group of neonates whose conjunctivitis did not resolve after neomycin treatment. In addition they found gonococci in $20 \%$ of this group of patients.

An authoritative opinion (British Medical Journal, 1974) suggests that gonococcal conjunctivitis occurs at a rate of one case per year in a large maternity unit and that the incidence of chlamydial infection is of a similar order. Dunlop (1975), however, claims that chlamydiae cause neonatal conjunctivitis at least five times more often than gonococci in the East End of London. At Northwick Park Hospital only 1 case of gonococcal neonatal conjunctivitis has been diagnosed in three years, and chlamydiae have been isolated from 6 neonates with persistent conjunctivitis at the hospital, although this is not a routine diagnostic procedure. This infrequent isolation of chlamydiae is in keeping with the failure to isolate them in the present study. In addition chlamydiae are insensitive to neomycin, but no case of neomycin-resistant conjunctivitis occurred. It seems reasonable to conlude, therefore, that chlamydial infection is a rare cause of neonatal conjunctivitis in the Harrow population.

Ureaplasmas were isolated from only 4 babies with conjunctivitis and from 2 control babies, the results suggesting no association between the presence of ureaplasmas on the conjunctivae and signs of conjunctivitis. There is, however, evidence of varying pathogenicity among bovine strains of ureaplasmas (Gourlay et al., 1972) and it has been suggested that some strains of human ureaplasmas cause disease in the male urogenital tract while others are harmless commensals (Prentice et al., 
1976). In view of this and the few ureaplasmapositive cases seen in this study, the results do not eliminate ureaplasmas as aetiological agents of a small proportion of cases of neonatal conjunctivitis.

Since the micro-organisms mentioned above do not seem to be a main cause of conjunctivitis in the Harrow population, and perhaps other populations too, there arises the question of which other microorganisms may be implicated in the disease. The isolation of Staph. aureus, viridans Streptococcus, and $E$. coli was statistically related to the presence of conjunctivitis, and in these cases we presume that the bacteria are pathogenic. Staph. epidermidis was not considered to be involved in the disease, since it was isolated at least as frequently from unaffected eyes. Furthermore no anaerobic bacteria were isolated from 63 patients or equal numbers of control infants, so there is no evidence that vaginal anaerobic bacteria can survive on the neonates' conjunctivae. Similarly, Trichomonas vaginalis was not seen in specimens from 32 affected infants, a finding which is in keeping with the negative observations of Thygeson (1971). Although trichomonads were observed by Novotný (1973) in 2 cases of neonatal conjunctivitis, it is not known whether they caused the disease.

No micro-organism was isolated from $53.5 \%$ of eyes with conjunctivitis, and only Staph. epidermidis was isolated from a further $10 \%$. The fact that cultures of specimens taken from infants with signs of conjunctivitis on the first day of life were uniformly negative suggests the possibility of chemical irritation as a cause in these cases. In others chemical irritation may also be a cause, but unidentified microbial infection, either bacterial or viral, cannot be ruled out. It is unlikely that herpes simplex virus is a cause, particularly in the absence of generalised disease (Nahmias and Hagler, 1972), since it is rarely found in the cervix in pregnancy. In view of this, and because there was insufficient conjunctival exudate to increase the number of specimens taken, culture for this virus was not attempted in this study. Nevertheless, it is possible that another virus producing a brief self-limiting disease is involved in some of the cases. Although it seems less likely that an undetected neomycin-sensitive organism is involved, the presence of such an agent may be confirmed or dismissed by a controlled study of the effect of neomycin therapy on the signs of disease.

We are grateful to the consultant paediatricians Dr M. M. Liberman and Dr H. B. Valman; Miss V. G. L. White; the nursing staff of the Maternity Unit and the domiciliary midwives. We also thank Mr R. Evans, the staff of the Microbiology Depart- ment at Northwick Park Hospital, and Dr K. McPherson, Mr M. J. R. Healy, and Dr I. D. Hill in the Division of Computing and Statistics.

\section{References}

Armstrong, J. H., Zacarias, F., and Rein, M. F. (1976). Ophthalmia neonatorum: a chart review. Pediatrics, 57, 884-892.

Bowie, W. R., Alexander, E. R., Floyd, J. F., Holmes, J., Miller, Y., and Holmes, K. K. (1976). Differential response of chlamydial and ureaplasma-associated urethritis to sulphafurazole (sulfisoxazole) and aminocyclitols. Lancet, 2, 1276-1278.

British Medical Journal (1974). Sticky eye in the newborn. 4, 222.

Burns, D. C. MacD., Darougar, S., Thin, R. N., Lothian, L., and Nicol, C. S. (1975). Isolation of Chlamydia from women attending a clinic for sexually transmitted disease. British Journal of Venereal Diseases, 51, 314-318.

Chiang, W. T., Alexander, E. R., Wei, P. Y., and Fresh, J. W. (1968). Genital infection with TRIC agents in Taiwan. American Journal of Obstetrics and Gynecology, 100, 422-431.

Darougar, S., Dwyer, R. St. C., Treharne, J. D., Harper, I. A., Garland, J. A., and Jones, B. R. (1970). A comparison of laboratory methods of diagnosis of chlamydial infection. Trachoma and Related Disorders, pp. 445-460. Edited by R. L. Nichols. Excerpta Medica: Amsterdam.

deLouvois, J., Stanley, V. C., Hurley, R., Jones, J. B., and Foulkes, J. E. B. (1975). Microbial ecology of the female lower genital tract during pregnancy. Postgraduate Medical Journal, 51, 156-160.

Dunlop, E. M. C., Al-Hussaini, M. K., Garland, J. A., Treharne, J. D., Harper, I. A., and Jones, B. R. (1965). Infection of urethra by TRIC agent in men presenting because of 'non-specific' urethritis. Lancet, 1, 1125-1128.

Dunlop, E. M. C. (1975). Recent advances in sexuallytransmitted diseases, p. 290 . Edited by R. S. Morton and J. R. W. Harris. Churchill Livingstone: London.

Foy, H. M., Kenny, G. E., Levinsohn, E. M., and Grayston, J. T. (1970). Acquisition of mycoplasmata and T-strains during infancy. The Journal of Infectious Diseases, 121, 579-587.

Freedman, A., Al-Hussaini, M. K., Dunlop, E. M. C., Emarah, M. H. M., Garland, J. A., Harper, I. A., Jones, B. R., Race, J. W., du Toit, M. S., Treharne, J. D., and Wright, D. J. M. (1966). Infection by TRIC agent and other members of the Bedsonia group, with a note on Reiter's disease. II. Ophthalmia neonatorum due to TRIC agent. Transactions of the Ophthalmological Societies of the United Kingdom, 86, 313-320.

Gordon, F. B., Harper, I. A., Quan, A. L., Treharne, J. D., Dwyer, R. St. C., and Garland, J. A. (1969). Detection of Chlamydia (Bedsonia) in certain infections of man. I. Laboratory procedures: comparison of yolk sac and cell culture for detection and isolation. The Journal of Infectious Diseases, 120, 451-461.

Gourlay, R. N., Howard, C. J., and Brownlie, J. (1972). The production of mastitis in cows by the intramammary inoculation of T-mycoplasmas. The Journal of Hygiene (Cambridge), 70, 511-521.

Hansman, D. (1969). Inclusion conjunctivitis. The Medical Journal of Australia, 1, 151-153.

Hobson, D., Johnson, F. W. A., Rees, E., and Tait, I. A. (1976). Chlamydia trachomatis isolations in Liverpool. Communicable Disease Report, 75, 51-52.

Hurley, R. (1966). Epidemic conjunctivitis in the newborn associated with coagulase-negative staphylococci. Journal 
of Obstetrics and Gynaecology British Commonwealth, 73, 990-992.

Jones, D. M., and Tobin, B. (1968). Neonatal eye infections due to Mycoplasma hominis. British Medical Journal, 3, 467-468.

Klein, J. O., Buckland, D., and Finland, M. (1969). Colonization of newborn infants by mycoplasmas. The New England Journal of Medicine, 280, 1025-1030.

Nahmias, A. J., and Hagler, W. S. (1972). Ocular manifestations of herpes simplex in the newborn (neonatal ocular herpes). International Ophthalmology Clinics, 12, 191-213.

Novotný, L. (1973). Trichomonas vaginalis as an agent in oculogenital conjunctivitis. Ceskoslovenska Oftalmologie, 29, 292-294.

Oriel, J. D., Powis, P. A., Reeve, P., Miller, A., and Nicol, C. S. (1974). Chlamydial infections of the cervix. British Journal of Venereal Diseases, 50, 11-16.

Prentice, M. J., Taylor-Robinson, D., and Csonka, G. W. (1976). Non-specific urethritis. A placebo-controlled trial of minocycline in conjunction with laboratory investigations. British Journal of Venereal Diseases, 52, 269-275.

Rees, E., and Hobson, D. (1974). Sticky eye in the newborn. British Medical Journal, 4, 656-657.

Schachter, J., Hanna, L., Hill, E. C., Massad, S., Sheppard, C. W., Conte, J. E. (Jr.), Cohen, S. N., and Meyer, K. F. (1975). Are chlamydial infections the most prevalent venereal disease? Journal of the American Medical Association, 231, 1252-1255.

Sowa, S., Sowa, J., and Collier, L. H. (1968). Investigation of neonatal conjunctivitis in the Gambia. Lancet, 2, 243-247.

Taylor-Robinson, D., Csonka, G. W., and Prentice, M. J. (1977). Human intra-urethral inoculation of ureaplasmas. Quarterly Journal of Medicine (In press).

Thygeson, P. (1971). Historical review of oculogenital disease. American Journal of Ophthalmology, 71, 975-985.

Watson, P. G., and Gairdner, D. (1968). TRIC agent as a cause of neonatal eye sepsis. British Medical Journal, 3, 527-528. 